\title{
Specific Formation of Cyclic Tetramer in Oligomerization of (R)-tert-Butyloxirane with Boron Trifluoride Etherate, and NMR and ORD Studies of the Cyclic Tetramer
}

\author{
Akira Sato, Tsuneo Hirano, Masahiro Suga, and Teiji Tsuruta \\ Department of Synthetic Chemistry, Faculty of Engineering, \\ University of Tokyo, Hongo, Bunkyo-ku, Tokyo 113, Japan.
}

(Received December 10, 1976)

\begin{abstract}
The reaction of (R)-tert-butyloxirane with boron trifluoride etherate as catalyst results in the specific formation of a cyclic tetramer, (2R,5R,8R,11R)-2,5,8,11tetra-tert-butyl-1,4,7,10-tetraoxacyclododecane, in high yield. The specificity of the reaction can be ascribed to steric requirements caused by the bulky tert-butyl group. Every monomeric unit, $-\left(\mathrm{O}-\mathrm{CH}\left(\right.\right.$ tert $\left.\left.-\mathrm{C}_{4} \mathrm{H}_{9}\right)-\mathrm{CH}_{2}-\right)$, in the cyclic tetramer preferably takes a $G^{+} G^{+} T$ conformation, in contrast to the $G^{+} G^{-} T$ conformation in the corresponding linear polymer. The ORD spectra of the cyclic tetramer are nearly the same in cyclohexane, in benzene, and in chloroform. The lack of solvent effect upon the ORD spectra may be explained in terms of the structure of the cyclic tetramer, in which the ether oxygen atoms wholly surrounded by the hydrocarbon parts of the cyclic tetramer are barely accessible to the solvent molecules.
\end{abstract}

KEY WORDS Oxirane / (R)-tert-Butyloxirane / Boron Trifluoride Etherate / Cationic Oligomerization / Cyclic Tetramer / (2R,5R,8R, 11R)-2,5,8,11-Tetra-tert-butyl-1,4,7,10-tetraoxacyclododecane / Crown Ether / NMR / ORD / Conformation /

We have recently reported that the bulky substituent group in tert-butyloxirane ( $t$-Bu-Oxir) exhibits unique features in the polymerization process $^{1,2}$ as well as in the properties of the polymer formed. ${ }^{3,4}$ The stereoselective behavior observed in the anionic polymerization of $t-\mathrm{Bu}-$ Oxir initiated by potassium tert-butoxide was interpreted in terms of stereoregulation due to interaction among the bulky substituents in the growing chain and in the incoming monomer. ${ }^{1,2}$ Our studies of the optical rotatory dispersion ${ }^{3}$ and the segmental motion ${ }^{4}$ of poly(alkyloxirane)s also showed that the main chain of $\operatorname{poly}((\mathbf{R})$ tert-butyloxirane) (poly ((R)-t-Bu-Oxir)) is less flexible, due to the bulky tert-butyl group, than and that of that of poly((S)-isopropyloxirane) poly((R)-methyloxirane).

In contrast to anionic polymerization, cationic polymerization of oxiranes with Lewis acid initiators has been known to produce various cyclic oligomers along with linear polymers. ${ }^{5}$ For instance, twenty-two isomers of twelve- membered cyclic tetramers were obtained in $30-40-\%$ yield by treating (RS)-methyloxirane with triethyloxonium tetrafluoroborate or boron trifluoride. ${ }^{6,7}$

Thus, expecting a specific effect of the bulky tert-butyl side group, we tried the cationic polymerization of $(\mathbf{R})$ - $t$-Bu-Oxir with boron trifluoride etherate $\left(\mathrm{BF}_{3} \cdot \mathrm{OEt}_{2}\right)$. In contrast to the case of methyloxirane, ${ }^{6,7}(\mathrm{R})-t$-Bu-Oxir produced cyclic tetramer of one type in high yield. We report here the specific formation of cyclic tetramer from (R)-t-Bu-Oxir, and the conformation and ORD properties of the cyclic tetramer obtained.

\section{EXPERIMENTAL}

\section{Materials}

Boron trifluoride etherate $\left(\mathrm{BF}_{3} \cdot \mathrm{OEt}_{2}\right)$ and solvents were used after distillation under dry nitrogen atmosphere.

$(R)$-tert-Butyloxirane $((R)-t-B u-O x i r)$ was pre- 
pared according to the following scheme, in a way similar to that reported by Sepulchre, et al.: ${ }^{8}$<smiles>CCCC(=O)CCCCCC(=O)CC(Br)C(O)OCC</smiles>

(1)

(2)

(3)

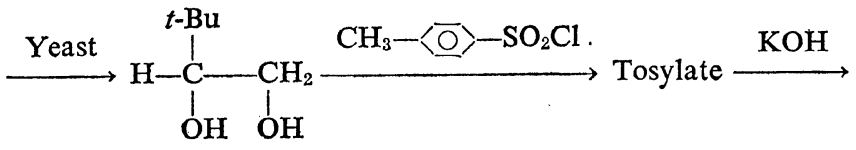

(4)

1-Bromo-3,3-dimethyl-2-butanone (2) was prepared from 3,3-dimethyl-2-butanone (1) by treating it with bromine: yield $70 \%$; bp $53^{\circ} \mathrm{C}$ (6 $\mathrm{mmHg})$.

1-Hydroxy-3,3-dimethyl-2-butanone (3) was prepared from 2 by the method of Levene, et al.: ${ }^{9}$ yield $70 \%$; bp $69^{\circ} \mathrm{C}(38 \mathrm{mmHg})$.

$(R)$-3,3-dimethyl-1,2-butanediol (4). To a mixture of $1 \mathrm{~kg}$ of baker's yeast and $1 \mathrm{~kg}$ of sugar in $10 l$ of water, $3(150 \mathrm{~g})$ was added, and the mixture was kept for three days at $38^{\circ} \mathrm{C}$ for fermentation. The reaction mixture was concentrated by evaporation under reduced pressure at $50^{\circ} \mathrm{C}$, and the oily residue was extracted with methanol-ether mixture in a large mortar. The supernatant organic solution separated by centrifugation was evaporated, and 4 was distilled from the residue: yield $35 \%$; bp $92^{\circ} \mathrm{C}$ $(8 \mathrm{mmHg}) ;[\alpha]_{\mathrm{D}}^{25}-27.6 \mathrm{deg} \mathrm{cm}^{2} / 10 \mathrm{~g}(c) 2.39 \mathrm{~g} / \mathrm{d} l$ in chloroform).

(R)-1-Tosyloxy-3,3-dimethyl-2-butanol (5) was prepared by tosylation of 4 with $p$-toluenesulfonyl chloride in pyridine solution according to Berti, et $a .^{10} 5$ was recrystallized from dichloromethane: yield $72 \% ; \mathrm{mp} 47.5^{\circ} \mathrm{C} ;[\alpha]_{\mathrm{D}}^{25}-26.3$ $\operatorname{deg} \mathrm{cm}^{2} / 10 \mathrm{~g}$ (c $1.31 \mathrm{~g} / \mathrm{d} l$ in dichloromethane).

$(R)$-tert-Butyloxirane $((R)-t$-Bu-Oxir) (6). Dropwise addition of 5 to an aqueous solution of potassium hydroxide $(160 \mathrm{~g} \mathrm{KOH}$ in $20 \mathrm{ml}$ of $\mathrm{H}_{2} \mathrm{O}$ ) at $60^{\circ} \mathrm{C}$ spontaneously produced a distillate, which was washed with $20-\% \mathrm{NaCl}$ aqueous solution and dried over $\mathrm{KOH}$ pellets. The crude (R)-t-Bu-Oxir was further purified by refluxing over $\mathrm{KOH}$ pellets and distilled at $96.5^{\circ} \mathrm{C}$. (R)-t-Bu-Oxir was then dried by refluxing over calcium hydride and lithium aluminum hydride and distilled again at $96.5^{\circ} \mathrm{C}$ under dry

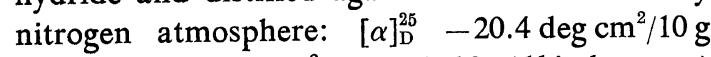
(neat), $-18.1 \mathrm{deg} \mathrm{cm} / 10 \mathrm{~g}$ (c1.83 $\mathrm{g} / \mathrm{d} l$ in benzene) hexane). $\mathrm{H}, 12.45$. products): $\mathrm{mp} 40-45^{\circ} \mathrm{C}$.
(5)<smiles>CCCCCCCCCCC</smiles>

(6)

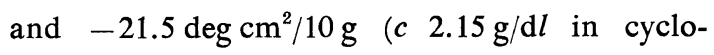

$(R S)$-tert-Butyloxirane $\quad((R S)-t-B u-O x i r)^{11}$ was prepared from 2 through reduction with sodium borohydride followed by ring closure in concentrated $\mathrm{KOH}$ aqueous solution.

(S)-Isopropyloxirane ( $(S)$-iPr-Oxir) was prepared by the method of Tsuji, et al.: ${ }^{12}[\alpha]_{\mathrm{D}}^{25} 4.83 \mathrm{deg}$ $\mathrm{cm}^{2} / 10 \mathrm{~g}$ (c $2.43 \mathrm{~g} / \mathrm{d} l$ in cyclohexane).

\section{Reaction and Analysis of Products}

Oligomerization with $\mathrm{BF}_{3} \cdot \mathrm{OEt}_{2}$

(i) (R)-t-Bu-Oxir. Dropwise addition of benzene solution of $\mathrm{BF}_{3} \cdot \mathrm{OEt}_{2}\left(0.83 \mathrm{mmol}\right.$ of $\mathrm{BF}_{3}$. $\mathrm{OEt}_{2}$ in $5 \mathrm{ml}$ of benzene) to the benzene solution of (R)-t-Bu-Oxir (20 mmol in $30 \mathrm{~m} l$ of benzene) at $0^{\circ} \mathrm{C}$ under dry nitrogen atmosphere induced a spontaneous exothermic reaction. After one day, the reaction mixture was washed successively with $\mathrm{H}_{2} \mathrm{O}, \mathrm{NaHCO}_{3}$ aqueous solution, and $\mathrm{H}_{2} \mathrm{O}$, and then submitted to freeze-drying to remove the benzene and the unreacted monomer. The yield of a colorless solid residue was 95\%. Repeated recrystallizations of the residue from methanol gave colorless needle crystals in $40 \%$ yield, based on the crude solid residue: $\mathrm{mp} 168 \pm 0.1^{\circ} \mathrm{C} ;[\alpha]_{\mathrm{D}}^{25} 50.8 \mathrm{deg} \mathrm{cm}^{2} / 10 \mathrm{~g}$ (c $1.10 \mathrm{~g} / \mathrm{d} l$ in cyclohexane) and $53.8 \mathrm{deg} \mathrm{cm} / 10$ $\mathrm{g}$ (c $1.28 \mathrm{~g} / \mathrm{d} l$ in benzene).

Anal. Calcd for cyclic tetramer of (R)-t-BuOxir: C, 71.90; H, 12.07. Found: C, 71.91;

(ii) (RS)-t-Bu-Oxir. (RS)-t-Bu-Oxir was reacted with $\mathrm{BF}_{3} \cdot \mathrm{OEt}_{2}$ under the same conditions as those of (R)-t-Bu-Oxir: yield $85 \%$. A methanol solution of the oligomerization products, upon cooling, gave a small amount of precipitates (12\% yield based on the crude

(iii) (S)-iPr-Oxir. (S)-iPr-Oxir was reacted 
with $\mathrm{BF}_{3} \cdot \mathrm{OEt}_{2}\left(4.2 \mathrm{~mol} \%\right.$ to (S)-iPr-Oxir) at $0^{\circ} \mathrm{C}$ in benzene. An oily product (yield 65\%) failed to give crystals even after purification.

Oligomerization of $(R)-t-B u-O x i r$ in Microscale. A deuterated benzene solution of a small amount of $(\mathrm{R})$ - $t$-Bu-Oxir was mixed with $\mathrm{BF}_{3} \cdot \mathrm{OEt}_{2}(6$ mol\% to (R)-t-Bu-Oxir) and sealed in a $10-\mathrm{mm} \phi$ NMR tube for ${ }^{13} \mathrm{C}$ NMR measurement.

Reaction of (R)-t-Bu-Oxir with Alcohol. (R)-tBu-Oxir (3.4 mmol) was reacted with tert-butyl alcohol $(3.4 \mathrm{mmol})$ in the presence of $\mathrm{BF}_{3} \cdot \mathrm{OEt}_{2}$ $(0.8 \mathrm{mmol})$ in deuterated benzene $(3 \mathrm{~m} l)$ at $0^{\circ} \mathrm{C}$. The reaction mixture was successively washed with $\mathrm{H}_{2} \mathrm{O}, \mathrm{NaHCO}_{3}$ aqueous solution, and $\mathrm{HO}_{2}$. After drying over $\mathrm{MgSO}_{4}$, the organic solution was submitted to ${ }^{13} \mathrm{C}$ NMR measurement. The reaction of (R)-t-Bu-Oxir with methyl alcohol in the presence of $\mathrm{BF}_{3} \cdot \mathrm{OEt}_{2}$ was carried out under the same conditions as those for tertbutyl alcohol.

\section{Measurements}

IR Spectra. IR spectra of chloroform solutions were measured in the $\mathrm{KBr}$ cell on a Hitachi EPI-G3 spectrometer.

Molecular Weight. The molecular weight was determined by vapor pressure osmometry in benzene at $37^{\circ} \mathrm{C}$ on a Mechrolab Osmometer Model 301A.

NMR. ${ }^{1} \mathrm{H}$ NMR spectra were recorded at 99.55 MHz on a Japan Electron Optics Laboratory Model PS-100 with TMS as internal standard. Pulsed Fourier transform ${ }^{13} \mathrm{C}$ NMR spectra were recorded at $25.03 \mathrm{MHz}$ on a Japan Electron Optics Laboratory Model PFT-100. The protons were decoupled from carbon-13 with a random noise-modulated decoupler: pulse width $45^{\circ}$, pulse repetition time $4-5 \mathrm{sec}$, and the number of accumulation 700-1000. All the chemical shifts are given in $\mathrm{ppm}$ with plus values for downfield shift ( $\delta$ scale) from the internal standard TMS.

\section{RESULTS AND DISCUSSION}

\section{Specific Formation of Cyclic Tetramer}

In the anionic polymerization of (R)-t-Bu-Oxir, a remarkable effect of the bulky side group was observed in the asymmetric selection of the monomer at the propagation stage. ${ }^{1,2}$ Analogously, a significant effect of the bulky tert-butyl group was also expected in the cationic polymerization.

In contrast to the various products in minor yields from cationic oligomerization of methyloxirane, ${ }^{6,7}$ the reaction system of (R)-t-Bu-Oxir with $\mathrm{BF}_{3} \cdot \mathrm{OEt}_{2}$ as catalyst (4 mol\% to (R)-t-BuOxir) in benzene gave a colorless crystal compound (7) in high yield (65\% or more). This crystal compound, which has a sharp melting point at $168 \pm 0.1{ }^{\circ} \mathrm{C}$, was concluded to be a tetramer of (R)-t-Bu-Oxir, because the molecular weight was found to be $400-410$ by vapor pressure osmometry and because the parent ion peak in the mass spectrum was at $400 \mathrm{~m} / e$. In the infrared spectrum, shown in Figure 1, the stretching absorption assignable to the ether $\mathrm{C}-\mathrm{O}$ bond was observed at $1000-1150 \mathrm{~cm}^{-1}$, but none of the characteristic absorptions of the hydroxyl group expected for the terminal

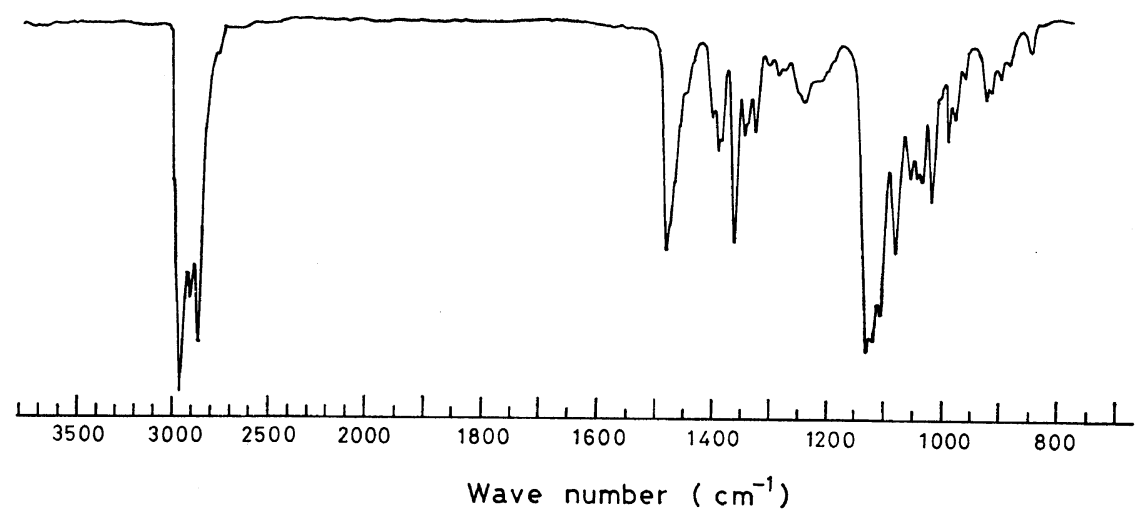

Figure 1. IR spectrum of the cyclic tetramer of (R)-tert-butyloxirane in chloroform. 
A. Sato, T. Hirano, M. Suga, and T. Tsuruta

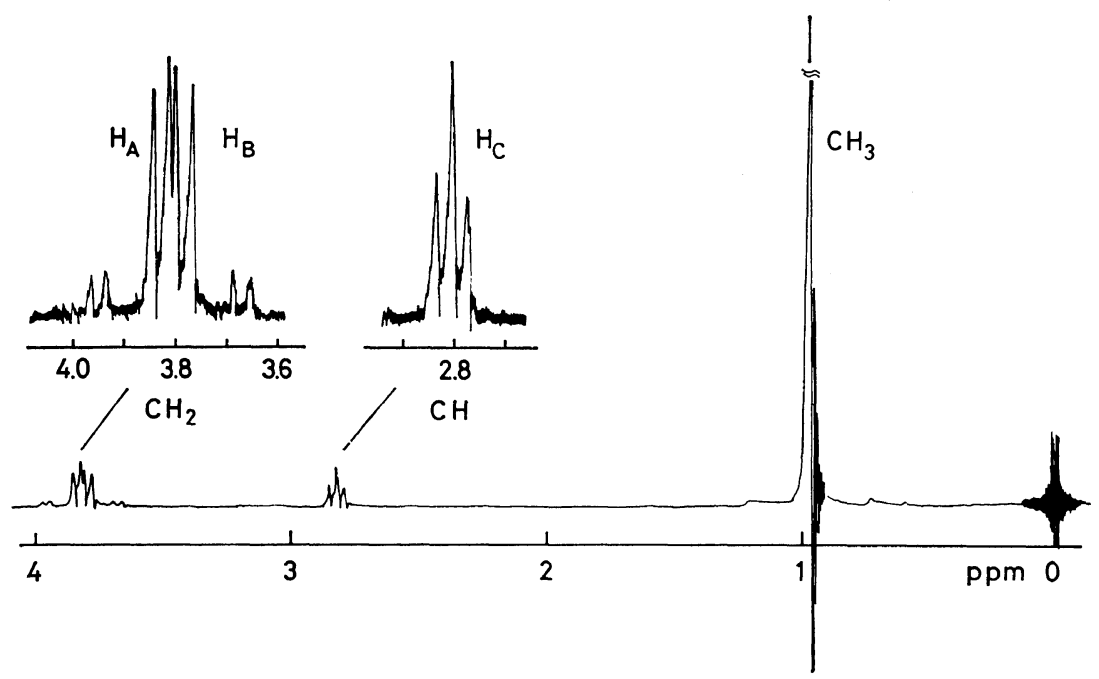

Figure 2. ${ }^{1} \mathrm{H}$ NMR spectrum of the cyclic tetramer of $(\mathbf{R})$-tert-butyloxirane in deuterated chloroform solution $(4 \mathrm{~g} / \mathrm{d} l)$ at $30^{\circ} \mathrm{C}, 99.55 \mathrm{MHz}$. $\mathrm{CH}_{3}$ denotes the methyl protons in the tert-butyl side group. $\mathrm{H}_{\mathrm{A}}, \mathrm{H}_{\mathrm{B}}$, and $\mathrm{H}_{\mathrm{C}}$ denote the protons in the main chain. The assignments are given in the text and in Figure 5.

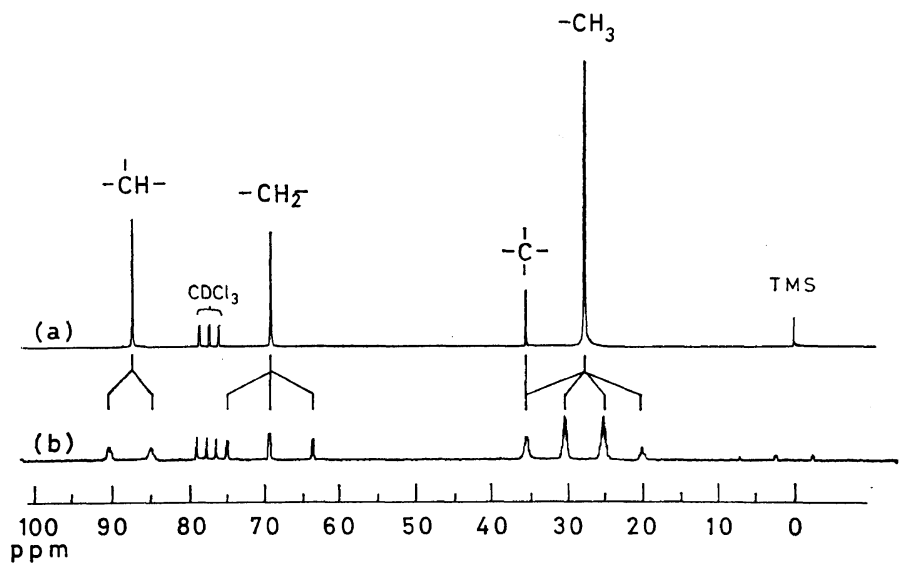

Figure 3. Pulsed Fourier transform ${ }^{13} \mathrm{C}$ NMR spectra $(25.03 \mathrm{MHz})$ of the cyclic tetramer of (R)-tert-butyloxirane in the deuterated chloroform solution $(10 \mathrm{~g} / \mathrm{d} l)$ at $50^{\circ} \mathrm{C}$ : (a), proton completely decoupled spectrum; (b), proton gated-decoupled spectrum.

group of a linear oligomer or polymer was observed. These results indicate that the crystal compound is a cyclic tetramer of (R)-t-Bu-Oxir. Further structural studies of this tetramer were carried out by ${ }^{1} \mathrm{H}$ and ${ }^{13} \mathrm{C}$ NMR spectroscopy.

As shown in Figure 2, ${ }^{1} \mathrm{H}$ NMR gave a sharp single line for the methyl group $(\delta 1.0 \mathrm{ppm})$ and an $\mathrm{ABC}$ coupling pattern for the $\mathrm{CH}-\mathrm{CH}_{2}$ protons $(\delta 2.8 \mathrm{ppm}$ and $3.6-4.0 \mathrm{ppm})$. No signal was observable for the hydroxyl proton. As Figure 3 shows, ${ }^{13} \mathrm{C}$ NMR gave a sharp single line for each carbon-13 atom of the methyl, quaternary, methylene, and methine groups. These results from ${ }^{1} \mathrm{H}$ and ${ }^{13} \mathrm{C}$ NMR studies indicate that the tetramer has no terminal unit and that each monomeric unit in this tetramer is configurationally equivalent, which is in agreement with the cyclic structure of the tetramer. 


\section{Cyclic Tetramer of (R)-tert-Butyloxirane}

The structural homogeneity discussed above means that the four asymmetric carbons in the cyclic tetramer (7) should all be in the (R) (or (S))-configuration. It should also be noted that the cyclic tetramer showed optical activity. Consequently, an extremely high degree of regioselectivity must be operative in the ring-opening process during the tetramer formation, though the site of bond scission cannot be determined from the results so far discussed.

Configuration around the Asymmetric Carbons in the Cyclic Tetramer (7)

As discussed in the preceding section, the oxirane ring of (R)-t-Bu-Oxir must have opened exclusively at either the $\mathrm{CH}-\mathrm{O}$ bond ( $\alpha$-opening) or the $\mathrm{CH}_{2}-\mathrm{O}$ bond ( $\beta$-opening) because the tetramer consists of four monomeric units possessing an identical configuration.

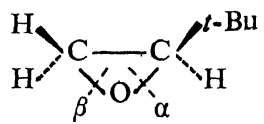

In order to obtain information on the site of ring opening, a model reaction of (R)-t-BuOxir with $\mathrm{BF}_{3} \cdot \mathrm{OEt}_{2}$ was carried out in the presence of tert-butyl alcohol or methyl alcohol in one to one mole ratio to the oxirane. After the unreacted alcohol was removed, the reaction mixture was examined by ${ }^{13} \mathrm{C}$ NMR. Results are shown in Figure 4. Assignments of the observed signals to the $\mathrm{CH}-, \mathrm{CH}_{2^{-}}$, and $\mathrm{CH}_{3}-$ carbons were confirmed with the gated decoupling technique. ${ }^{3}$ The signals at 74 and $89 \mathrm{ppm}$ in (d) and (e) are assigned to ${ }^{13} \mathrm{C}$ resonances of the $\mathrm{CH}_{2}-\mathrm{O}$ and $\mathrm{CH}-\mathrm{O}$ carbons, respectively, of the internal oxy(1-tert-butylethylene) unit, in comparison with the spectra of the linear polymer $((b))^{3}$ and oligomer $((c))^{13}$ obtained by anionic polymerization. Comparison of the spectra (d) and (e) with those of (R)-3,3dimethyl-1,2-butanediol (4) ((a)) and the linear oligomer ((c)) indicates that the signal at $78 \mathrm{ppm}$ is the signal of the terminal secondary alcoholic carbon $(-\stackrel{\prime}{C} \mathrm{H}-\mathrm{OH})$. The signals at 64 and 73 ppm are assigned to the $\left(\mathrm{CH}_{3}\right)_{3} \mathrm{C}-\mathrm{O}-\mathrm{CH}_{2}-$ and $\left(\mathrm{CH}_{3}\right)_{3} \mathrm{C}-\mathrm{O}-\mathrm{CH}_{2}-$ carbons, respectively, from comparison with spectrum (c). Although the $\mathrm{CH}_{2}-\mathrm{OH}$ signal of (R)-3,3-dimethyl-1,2-butanediol appears at $64 \mathrm{ppm}((\mathrm{a}))$, the signal at $64 \mathrm{ppm}$ seen in (d) cannot be assigned to that of the $\mathrm{CH}_{2}-\mathrm{OH}$, because the signal at $64 \mathrm{ppm}$ as well as that at $73 \mathrm{ppm}$ was not observed when methyl alcohol was used in place of tert-butyl alcohol in the model reaction (e).

These results mean that the reaction of (R)$t$-Bu-Oxir with $\mathrm{BF}_{3} \cdot \mathrm{OEt}_{2}$ in the presence of alcohol $(\mathrm{R}-\mathrm{OH})$ gave products that have both $\mathrm{HO}-\stackrel{\mathrm{C}}{\mathrm{C}}-$ and $-\mathrm{CH}_{2}-\mathrm{OR}$ end groups, but

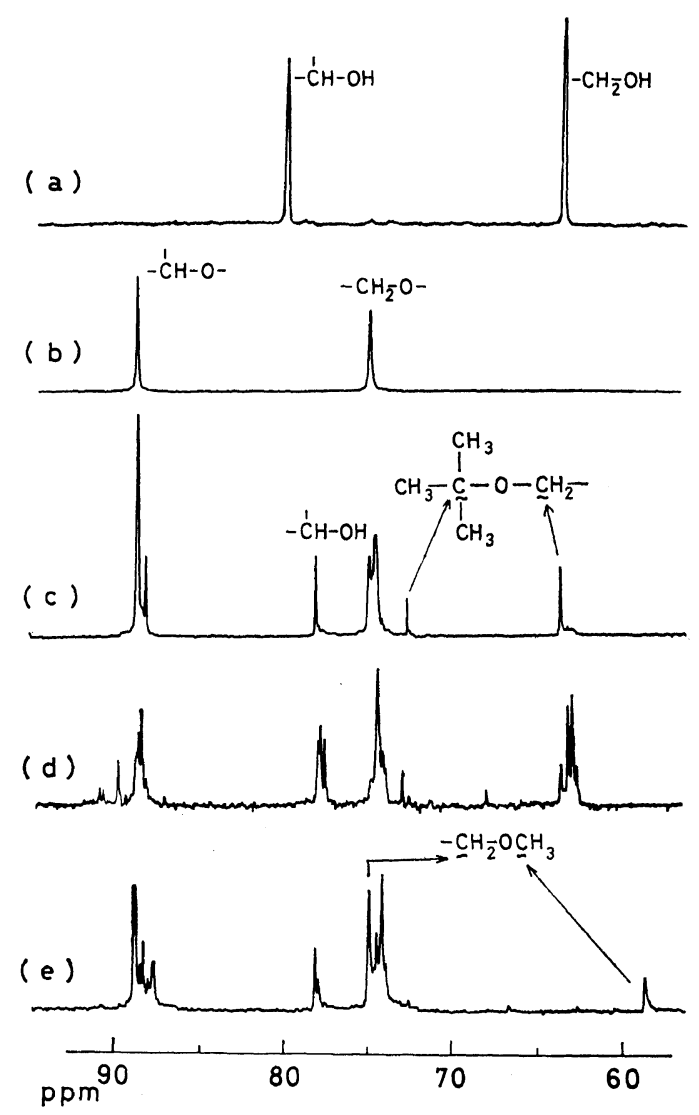

Figure 4. Pulsed Fourier transform ${ }^{13} \mathrm{C}$ NMR spectra $(25.03 \mathrm{MHz})$ of the various products from (R)-tert-butyloxirane in deuterated benzene (10-30 $\mathrm{g} / \mathrm{d} l$ ) at $50^{\circ} \mathrm{C}$ : (a), (R)-3,3-dimethyl-1,2-butanediol (4); (b), poly((R)-tert-butyloxirane) obtained by the polymerization with potassium tert-butoxide; (c), linear oligomer of (R)-tert-butyloxirane obtained by the reaction with potassium tert-butoxide; (d), products obtained by the reaction of (R)-tertbutyloxirane and tert-butyl alcohol in the presence of $\mathrm{BF}_{3} \cdot \mathrm{OEt}_{2} ;(\mathrm{e})$, products obtained by the reaction of (R)-tert-butyloxirane and methyl alcohol in the presence of $\mathrm{BF}_{3} \cdot \mathrm{OEt}_{2}$. 
not $\mathrm{HO}-\mathrm{CH}_{2}-$ and $-\stackrel{\mathrm{C}}{\mathrm{C}}-\mathrm{OR}$ end groups. From the results of the model reaction, it is most probable that the cyclic tetramerization of $(\mathrm{R})-t$-Bu-Oxir with $\mathrm{BF}_{3} \cdot \mathrm{OEt}_{2}$ catalyst proceeds under the cleavage of the $\mathrm{CH}_{2}-\mathrm{O}$ bonds $(\beta$ opening), although the reaction conditions of the model reaction were not completely the same as those of the tetramerization.

The $\beta$-opening of the alkyloxirane ring results in the retention of the configuration at the asymmetric carbon. Therefore, the configuration of all the asymmetric carbons of the cyclic tetramer from (R)-t-Bu-Oxir should be rectus $(\mathrm{R})$ so far as the assumption of $\beta$-opening is valid. The discussion hereafter is based on the tentative conclusion that the cyclic tetramer is $(2 R$, $5 \mathrm{R}, 8 \mathrm{R}, 11 \mathrm{R})-2,5,8,11$-tetra-tert-butyl-1, 4, 7, 10tetraoxacyclododecane.<smiles>CCCCCC(CCCC)OCC(CCCCC)OCC(CCCC)OCC(C)CC</smiles>

Conformation of the Cyclic Tetramer (7)

As was discussed in our previous studies of linear poly(alkyloxirane)s, ${ }^{3,12}$ a bulky side group reduces the number of the possible preferred conformations. Similarly, but under the more severe restriction of being cyclic, the tert-butyl group should play an important role in the conformation of the cyclic tetramer. Thus, the conformation of the cyclic tetramer (7) was studied by ${ }^{1} \mathrm{H}$ and ${ }^{13} \mathrm{C}$ NMR in comparison with the corresponding linear polymer, poly $((\mathbf{R})$-tertbutyloxirane) (poly((R)-t-Bu-Oxir)).

(i) ${ }^{1} H$ NMR. The ${ }^{1} \mathrm{H}$ NMR spectrum of the cyclic tetramer (7) in deuterated chloroform is shown in Figure 2. The methine-methylene part of the spectrum ( $\delta 2.8-4.0 \mathrm{ppm})$ was analyzed as an $A B C$ spin system with the aid of a LAOCN3 simulation program. ${ }^{14}$ The chemical shift and coupling constant values are listed in Table I. The signals around $\delta 2.8 \mathrm{ppm}$ were assigned to the methine proton $\mathrm{H}_{\mathrm{C}}$, and those around $\delta 3.6-$ $3.8 \mathrm{ppm}$ and $\delta 3.8-4.0 \mathrm{ppm}$ to $\mathrm{H}_{\mathrm{B}}$ and $\mathrm{H}_{\mathrm{A}}$
Table I. Chemical shifts and coupling constants for the cyclic tetramer, $(2 \mathrm{R}, 5 \mathrm{R}, 8 \mathrm{R}, 11 \mathrm{R})-2,5,8,11$ tetra-tert-butyl-1,4,7,10-tetraoxacyclododecane, in deuterated benzene and in deuterated chloroform at various temperatures; the assignments of A, B, and $\mathrm{C}$ protons are given in the text and in Figure 5.

\begin{tabular}{|c|c|c|c|c|c|c|c|}
\hline \multirow[t]{2}{*}{ Solvent } & \multirow{2}{*}{${ }_{{ }^{\circ} \mathrm{C}}^{\text {Temp }}$} & \multicolumn{3}{|c|}{$\begin{array}{c}\text { Chemical shift, }{ }^{\mathrm{a}} \\
\text { ppm }\end{array}$} & \multicolumn{3}{|c|}{$\begin{array}{l}\text { Coupling } \\
\text { constant, } \mathrm{Hz}\end{array}$} \\
\hline & & $\delta_{\mathrm{A}}$ & $\delta_{\mathrm{B}}$ & $\delta_{\mathrm{C}}$ & $\left|{ }^{3} J_{\mathrm{AB}}\right|$ & ${ }^{3} \boldsymbol{J}_{\mathrm{AC}}$ & $\left|{ }^{3} \boldsymbol{J}_{\mathrm{BC}}\right|$ \\
\hline \multirow[t]{3}{*}{$\mathrm{C}_{6} \mathrm{D}_{6}$} & 30 & 3.70 & 3.64 & 2.66 & 11.7 & 2.6 & 3.0 \\
\hline & 50 & 3.76 & 3.68 & 2.71 & 11.8 & 2.7 & 3.3 \\
\hline & 75 & 3.81 & 3.71 & 2.75 & 11.7 & 2.6 & 3.3 \\
\hline \multirow[t]{2}{*}{$\mathrm{CDCl}_{3}$} & 30 & 3.90 & 3.78 & 2.83 & 12.0 & 3.0 & 3.2 \\
\hline & 50 & 3.90 & 3.79 & 2.83 & 11.9 & 2.9 & 3.3 \\
\hline
\end{tabular}

a Chemical shift is given in ppm with plus value for downfield shift ( $\delta$ scale) from the internal standard TMS.
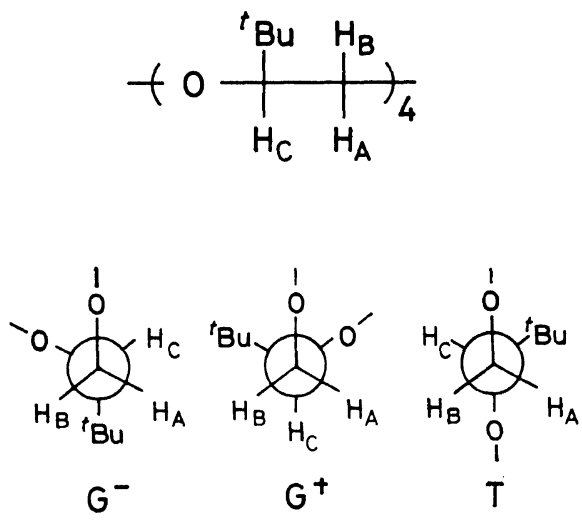

Figure 5. Configurational assignments of protons $\mathrm{H}_{\mathrm{A}}, \mathrm{H}_{B}$, and $\mathrm{H}_{\mathrm{C}}$, and three possible conformations around the main chain $\mathrm{CH}-\mathrm{CH}_{2}$ bond of the cyclic tetramer of $(\mathbf{R})$-tert-butyloxirane.

methylene protons, respectively; the Newman projection for the possible three conformers around the methylene-methine carbon bond and the Fischer projection for the constitutional unit are shown in Figure 5.

The assignments of $H_{A}$ and $H_{B}$ are made from the ${ }^{3} J$ 's and the deshielding effect of the ether oxygen atoms as follows. On the basis of the Karplus-type dependence of ${ }^{3} J$ on the dihedral angle, ${ }^{15}$ it was concluded that $\mathrm{H}_{\mathrm{C}}$ should come approximately on the bisection plane of $\mathrm{H}_{A}$ and $\mathrm{H}_{\mathrm{B}}$, because the observed ${ }^{3} J_{\mathrm{AC}}$ and ${ }^{3} J_{\mathrm{BC}}$ were both about $3 \mathrm{~Hz}$ (Table I). Therefore, the pre- 


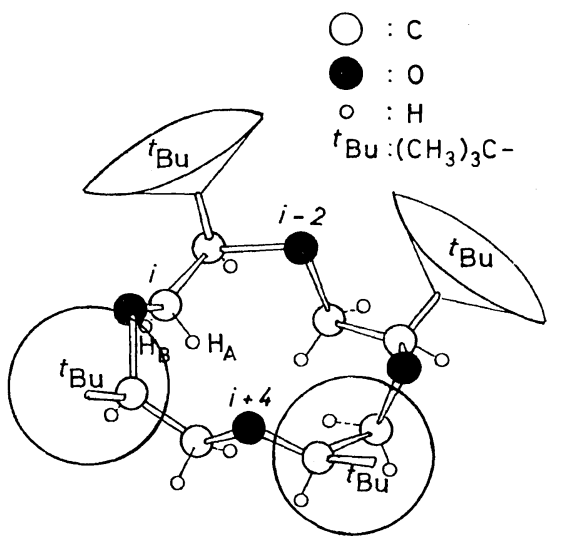

Figure 6. The most reasonable structure of the cyclic tetramer of ( $\mathbf{R})$-tert-butyloxirane; in this structure, all the oxygen atoms are wholly surrounded by the hydrocarbon parts of the cyclic tetramer.

dominant conformation around the main chain $\mathrm{CH}-\mathrm{CH}_{2}$ bond should be $\mathrm{G}^{+}$in Figure 5 .

The most probable spatial structure for the cyclic tetramer is the structure shown in Figure 6 , where the main chain of the tetramer predominantly takes a $G^{+} G^{+} T$ conformation:

$$
\left|\begin{array}{c}
t-\mathrm{Bu} \\
-\left(\mathrm{O}-\mathrm{CH}-\mathrm{CH}_{2}-{ }_{4}^{+}\right.
\end{array}\right|
$$

According to this structure, $\mathrm{H}_{\mathrm{A}}$ should be more deshielded than $\mathrm{H}_{\mathrm{B}}$ because two ether oxygen atoms $\mathrm{O}(i-2)$ and $\mathrm{O}(i+4)$ come closer to $\mathrm{H}_{\mathrm{A}}$ than to $\mathrm{H}_{\mathrm{B}}$ of the methylene group (i). ${ }^{*}$ Therefore, the lower field signal of the two observed methylene signals is assigned to the $H_{A}$ proton and the upper one to the $\mathrm{H}_{B}$ proton.

(ii) ${ }^{13} \mathrm{C} \mathrm{NMR}$. The ${ }^{13} \mathrm{C}$ NMR spectrum of the cyclic tetramer (7) in deuterated chloroform is shown in Figure 3. The methylene carbon-13 signal of the cyclic tetramer (7) appears at a higher magnetic field than that of the corresponding linear polymer, poly ((R)-t-Bu-Oxir), by $6.04 \mathrm{ppm}$ ( $c f$. Figure 4b). This difference in chemical shifts might be due to the difference

* An oxygen atom in close proximity to a proton causes a downfield shift: L. M. Jackman and $S$. Sternhell, "Applications of Nuclear Magnetic Resonance Spectroscopy in Organic Chemistry,', 2nd ed., Pergamon Press, Oxford, 1969, p 81. in the preferred conformations of the linear polymer $\left(G^{+} G^{-} T\right)$ and of the cyclic tetramer $\left(G^{+} G^{+} T\right)$. Under the conformation of $G^{+} G^{+} T$, methylene carbons in the cyclic tetramer (7) come close to each other (Figure 6). Thus, the observed higher field shift of the methylene carbon signal of the cyclic tetramer might be interpreted in terms of the steric compression shift, ${ }^{16}$ magnetic anisotropy effect, and some other factors.

Optical Rotatory Dispersion Spectra of the Cyclic Tetramer (7)

The ORD spectra of the cyclic tetramer (7) in benzene, in cyclohexane, and in chloroform are shown in Figure 7. In contrast to the corresponding linear poly(alkyloxirane)s, ${ }^{3,12}$ the ORD curve of the cyclic tetramer (7) in cyclohexane is exactly the same as that in benzene and very similar to that in chloroform.

According to our previous study of the ORD properties of linear poly(alkyloxirane)s, ${ }^{3,12}$ the different ORD behaviors in cyclohexane and in benzene can be ascribed to the different degree of interaction between solvent molecules and the main chain of the polyether, i.e., a smaller degree of interaction was observed in the poly(alkyloxirane) having the bulkier side group. The similarity of the ORD spectra of the cyclic tetramer (7) in benzene and in cyclohexane indicates the absence of an appreciable interaction of solvent molecules with the main chain. This

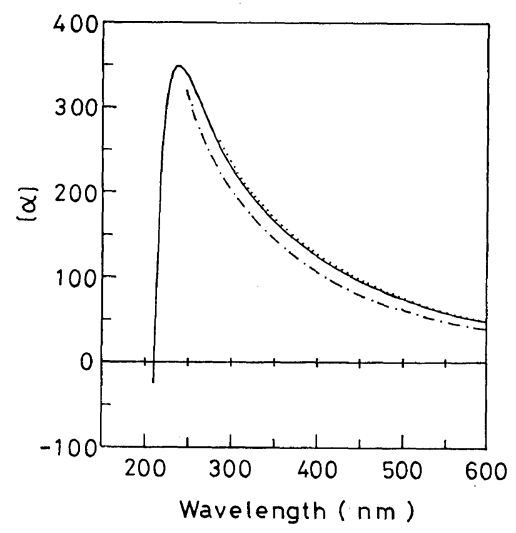

Figure 7. ORD spectra of the cyclic tetramer of (R)-tert-butyloxirane at $25^{\circ} \mathrm{C}$ : (-) in cyclohexane $(1.10 \mathrm{~g} / \mathrm{d} l) ;(\cdots)$ in benzene $(1.28 \mathrm{~g} / \mathrm{d} l)$; $(-\cdot--)$ in chloroform $(1.06 \mathrm{~g} / \mathrm{d} l)$. 
indication may be explained by the proposed structure of the cyclic tetramer (7) (Figure 6), where the ether oxygen atoms are insulated from the solvent by the bulky hydrocarbon groups.

Mode of Oligomer Distribution and Comparison with Those of Other Oxiranes

Oligomerization of several oxiranes in the presence of $\mathrm{BF}_{3} \cdot \mathrm{OEt}_{2}$ was studied from the point of oligomer distribution.

(i) (R)-t-Bu-Oxir. The oligomerization reaction was followed in a $10-\mathrm{mm} \phi \mathrm{NMR}$ tube by ${ }^{13} \mathrm{C}$ NMR measurement. The ${ }^{13} \mathrm{C}$ NMR signals of (R)-t-Bu-Oxir disappeared within a few minutes at room temperature under the experimental conditions given in the Experimental section above, and the area of each signal of the cyclic tetramer showed that about $68 \%$ of the reaction products was the cyclic tetramer (7). In addition to the signals of the cyclic tetramer (7), those of linear oligomers were found in the reaction system.

The gel-permeation chromatogram (GPC) of the crude colorless solid obtained from the reaction mixture showed three main peaks, one corresponding to the cyclic tetramer and the other two to higher molecular weight oligomers (pentamer and hexamer, judging from the molecular weight calibration with polystyrene) (Figure 8a). The peak area of the cyclic tetramer in GPC spectrum amounts to about $65 \%$ of all areas. After separation of the cyclic tetramer (7) from the crude colorless solid as methanolinsoluble precipitates (colorless crystals), the methanol-soluble part was further examined by GPC to determine the composition of the oligomers other than the cyclic tetramer. Seven peaks including the cyclic tetramer were observed in the GPC spectrum (Figure $8 b$ ). The molecular weight calibration with polystyrene indicates that these peaks correspond to the dimer...octamer sequence. No attempt was made, however, to determine whether these oligomers are linear or cyclic.

(ii) (RS)-tert-Butyloxirane ((RS)-t-Bu-Oxir). The GPC profile of the reaction products (Figure 9) was quite similar to that of the products from (R)- $t$-Bu-Oxir, and the infrared spectrum of the products shows a very weak $\nu_{\mathrm{OH}}$ absorption. Thus, the main reaction products from (RS)-t-Bu-Oxir were also cyclic tetramers.

The same separation procedure as that for the crude products from (R)- $t$-Bu-Oxir was applied for the reaction products from (RS)-t-BuOxir. In contrast, a relatively small amount of precipitates (about 12\% of the total product) was obtained as a methanol-insoluble fraction,
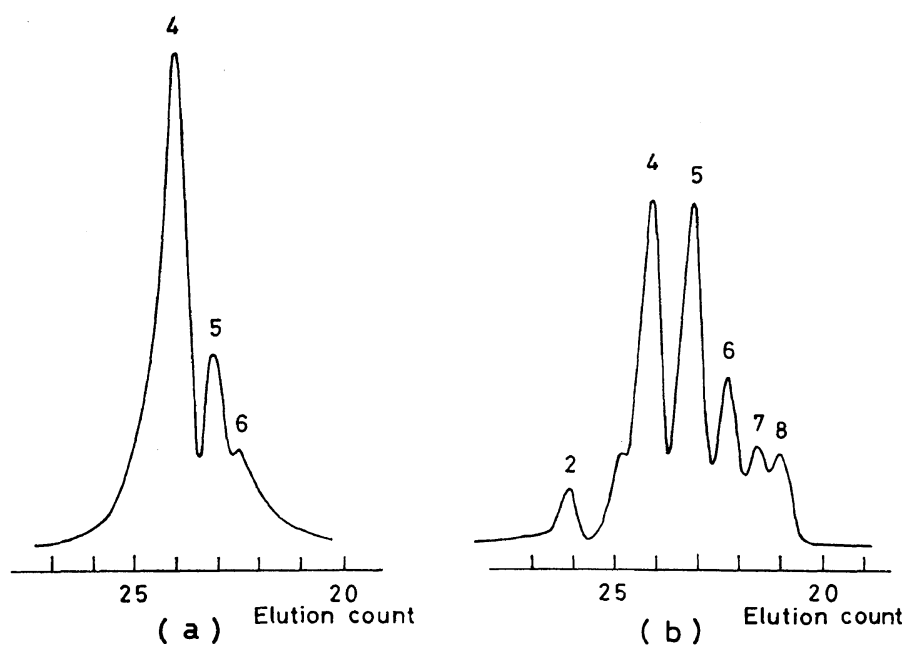

Figure 8. GPC spectra of the products obtained from the reaction of (R)tert-butyloxirane with $\mathrm{BF}_{3} \cdot \mathrm{OEt}_{2}$ as catalyst: (a), the crude product; (b), the methanol-soluble part. The number in the figures denotes the number of monomeric units in the oligomer (e.g., 4 for the tetramer). 
which gave a similar ${ }^{13} \mathrm{C}$ NMR spectrum to that of the crude products. It follows that methanol fractionation was not effective in this case. The difference from the oligomerization of $(\mathbf{R})-t-\mathrm{Bu}-$ Oxir is that the amount of the precipitates from methanol solution was small and that the melting point of the precipitates was not sharp. The

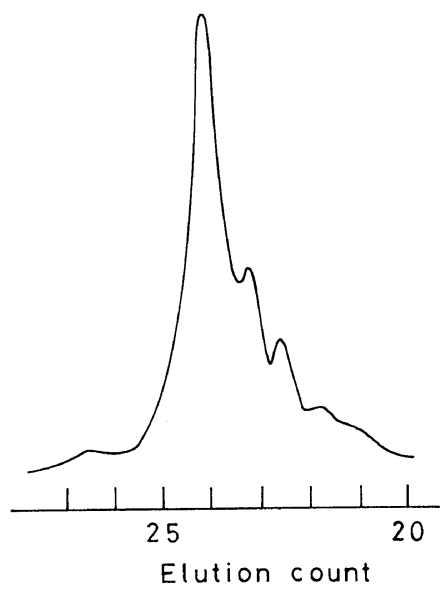

Figure 9. GPC spectrum of the crude products obtained from the reaction of (RS)-tert-butyloxirane with $\mathrm{BF}_{3} \cdot \mathrm{OEt}_{2}$ as catalyst. latter indicates that the precipitates should not be a pure cyclic tetramer of one type. Indeed, as is shown in Figure 10 , the ${ }^{13} \mathrm{C}$ NMR spectrum of the precipitates shows several peaks for each carbon, i.e., $\mathrm{CH}_{3}, \mathrm{C}, \mathrm{CH}_{2}$, and $\mathrm{CH}$, indicating that the precipitates contain several diastereoisomers in different combinations of $(\mathrm{R})$ - and (S)-residues in head-to-tail sequences. No specific stereoselection producing cyclic tetramers of a certains type such as (RRRR) plus (SSSS) or (RRSS) operated in this oligomerization where both (R)- and (S)-monomers were present.

(iii) (S)-isopropyloxirane $((S)$-iPr-Oxir). The reaction product was an oily compound which was found to contain at least seven major products of low molecular weight from gas-liquid chromatography and GPC measurements.

\section{CONCLUSION}

(1) Oligomerizations of (R)-t-Bu-Oxir, (RS)-tBu-Oxir, and (S)-iPr-Oxir were carried out with $\mathrm{BF}_{3} \cdot \mathrm{OEt}_{2}$ as catalyst. (R)-t-Bu-Oxir gave specifically a cyclic tetramer in high yield. (RS)-tBu-Oxir and (S)-iPr-Oxir, however, did not give a single pure product but gave several products

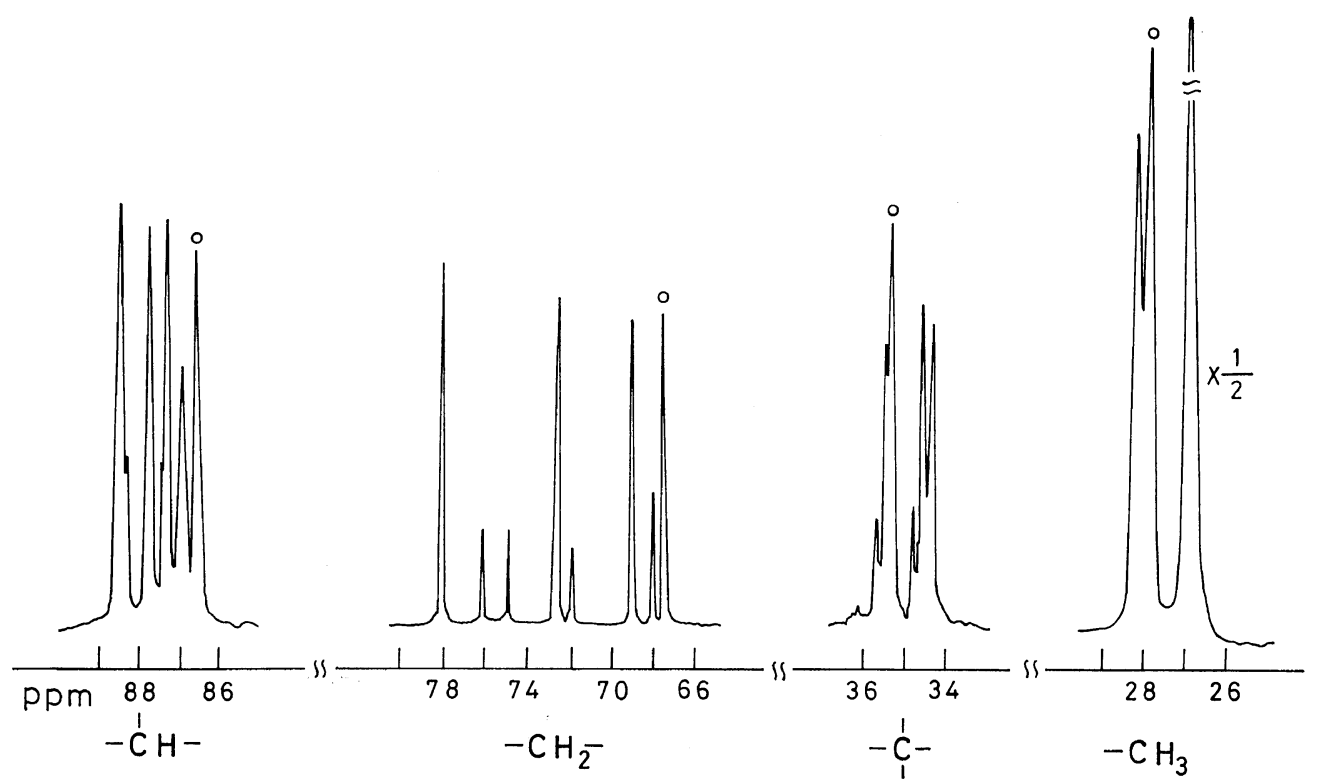

Figure 10. Pulsed Fourier transform ${ }^{13} \mathrm{C}$ NMR spectrum $(25.03 \mathrm{MHz})$ of the methanol-insoluble part of the products obtained from the reaction of (RS)-tert-butyloxirane with $\mathrm{BF}_{3} \cdot \mathrm{OEt}_{2}$ as catalyst; the spectrum was obtained in the deuterated benzene solution $(10-15 \mathrm{~g} / \mathrm{d} l)$ at $30^{\circ} \mathrm{C}$. The chemical shifts of the peaks marked by $\bigcirc$ are the same as those of the cyclic tetramer of (R)-tert-butyloxirane. 


\section{A. Sato, T. Hirano, M. Suga, and T. Tsuruta}

in minor yields. The specificity found in the oligomerization of (R)- $t$-Bu-Oxir should be ascribed to the effect of the bulkiness of the tertbutyl group, from comparison with our results of the oligomerization of (S)-iPr-Oxir and with those of (RS)-methyloxirane reported by Katnik, et $a .^{7}$

A similar specificity producing cyclic tetramers through the $\beta$-opening of the oxirane ring was suggested in the oligomerization of (RS)- $t$ Bu-Oxir, although several diastereoisomeric tetramers were obtained due to the presence of $(\mathbf{R})$ - and (S)-monomers in the reaction system.

(2) ${ }^{1} \mathrm{H}$ and ${ }^{13} \mathrm{C}$ NMR studies made clear that the cyclic tetramer takes a $\left(G^{+} G^{+} T\right)_{4}$ conformation in chloroform solution in contrast to the case of the corresponding linear polymer $\left(\left(G^{+} G^{-} T\right)_{n}\right)$.

(3) The optical rotatory dispersion spectra of the cyclic tetramer in cyclohexane, in benzene, and in chloroform were quite similar to each other. The lack of solvent effect on the ORD curve is explained by the unique structure of the cyclic tetramer, in which the interaction of the main chain oxygen atoms with the solvent molecules is hindered; i.e., the oxygen atoms are wholly surrounded by the hydrocarbon groups of the cyclic tetramer in the $G^{+} G^{+} T$ conformation and are barely accessible to the solvent molecules. This explanation is consistent with our previous conclusion that the differences in the solvent effect on the ORD spectra of linear poly(alkyloxirane)s can be ascribed to the different magnitudes of the interaction of the solvent molecules with the main chain of the polyethers. ${ }^{3,12}$

(4) The cyclic tetramer, since it is a kind of "Crown Ether," can be expected to form a complex with alkali metal ion. Indeed, the specific interactions with $\mathrm{Li}^{+}$and $\mathrm{Na}^{+}$were observed, by ${ }^{1} \mathrm{H}$ NMR, in the deuterated acetone solution. A detailed study of the cation-binding property of the cyclic tetramer will be reported elsewhere.

Note added in Proof

Remark on the assignments of $H_{A}$ and $H_{B}$ protons (Figure 5): The magnetic bond anisotropy effect of the tert-butyl group in the same monomeric unit contributes also to the deshielding of the $\mathrm{H}_{\mathrm{A}}$ proton and to the shielding of the $\mathrm{H}_{B}$ proton in the proposed structure of the cyclic tetramer shown in Figure 6 (cf. ref. 12, and H. M. McConnell, J. Chem. Phys., 27, 226 (1957)).

\section{REFERENCES}

1. A. Sato, T. Hirano, and T. Tsuruta, Makromol. Chem., 178, 609 (1977).

2. A. Sato, T. Hirano, and T. Tsuruta, ibid., 176, 1187 (1975).

3. A. Sato, T. Hirano, and T. Tsuruta, ibid., 177, 3059 (1976).

4. A. Sato, T. Hirano, and T. Tsuruta, to be published.

5. Y. Ishii and S. Sakai in "Ring-Opening Polymerization," K. C. Frisch and S. L. Reegen, Ed., Marcel Dekker, New York, N.Y., 1969, pp 13-109.

6. R. J. Kern, J. Org. Chem., 33, 388 (1968).

7. R. J. Katnik and J. Schaefer, ibid., 33, 384 (1968).

8. M. Sepulchre and A.-M. Sepulchre, Bull. Soc. Chim. Fr., 1973, 1164.

9. P. A. Levene and A. Walti, J. Biol. Chem., 68, 415 (1926).

10. G. Berti, F. Bottari, and B. Macchia, Ann. Chim. (Rome), 52, 1101 (1962).

11. S. J. Hurst and J. M. Bruce, J. Chem. Soc., 1963, 1321.

12. K. Tsuji, T. Hirano, and T. Tsuruta, Makromol. Chem. Suppl., 1, 55 (1975).

13. A. Sato, T. Hirano, and T. Tsuruta, to be published.

14. A. A. Bothner-By and S. M. Castellano in "Computer Programs for Chemistry," Vol. I, D. F. DeTar, Ed., Benjamin Inc., New York, N.Y., 1968, pp 10-53.

15. M. Karplus, J. Chem. Phys., 30, 11 (1959); J. Am. Chem. Soc., 85, 2870 (1963).

16. G. C. Levy and G. L. Nelson, "Carbon-13 Nuclear Magnetic Resonance for Organic Chemists," Wiley-Interscience, New York, N.Y., 1972, pp 24, 43. 